\title{
Utility of clinical parameters to identify HIV infection in infants below ten weeks of age in South Africa: a prospective cohort study
}

Heather B Jaspan ${ }^{1,2,3,9^{*}}$, Landon Myer ${ }^{4}$, Shabir A Madhi ${ }^{5}$, Avy Violari ${ }^{6}$, Diana M Gibb ${ }^{7}$, Wendy S Stevens ${ }^{8}$, Els Dobbels ${ }^{1}$ and Mark F Cotton ${ }^{1}$

\begin{abstract}
Background: As HIV-infected infants have high mortality, the World Health Organization now recommends initiating antiretroviral therapy as early as possible in the first year of life. However, in many settings, laboratory diagnosis of HIV in infants is not readily available. We aimed to develop a clinical algorithm for HIV presumptive diagnosis in infants $<10$ weeks old using screening data from the Children with HIV Early Antiretroviral therapy (CHER) study in South Africa.

HIV-infected and HIV-uninfected exposed infants $<10$ weeks of age were identified through Vertical Transmission Prevention programs. Clinical and laboratory data were systematically recorded, groups were compared using Kruskal-Wallis, analysis of variance (ANOVA), and Fisher's exact tests. Receiver Operating Characteristic (ROC) curves were compiled using combinations of clinical findings.

Results: 417 HIV-infected and 125 HIV-exposed, uninfected infants, median age 46 days (IQR 38-55), were included. The median CD4 percentage in HIV-infected infants was 34 (IQR 28-41)\%. HIV-infected infants had lower weightfor-age, more lymphadenopathy, oral thrush, and hepatomegaly than exposed uninfected infants (Adjusted Odds Ratio $0.51,8.8,5.6$ and 23.5 respectively; $p<0.001$ for all). Sensitivity of individual signs was low $(<20 \%)$ but specificity high (98-100\%). If any one of oral thrush, hepatomegaly, splenomegaly, lymphadenopathy, diaper dermatitis, weight $<50^{\text {th }}$ centile are present, sensitivity for HIV infection amongst HIV-exposed infants was $86 \%$. These algorithms performed similarly when used to predict severe immune suppression.

Conclusions: A combination of physical findings is helpful in identifying infants most likely to be HIV-infected. This may inform management algorithms and provide guidance for focused laboratory testing in some settings, and should be further validated in these settings and elsewhere.
\end{abstract}

\section{Background}

Identifying HIV infection in early infancy is an important challenge to effective paediatric HIV care and treatment. HIV-infected infants have a high mortality, particularly in heavily disease-burdened settings [1-6]. The Children with $\underline{\text { HIV E}}$ Early Antiretroviral Therapy (CHER) trial [4] recently showed that early antiretroviral therapy (ART), commenced at a median of 7 weeks of age was associated with a $75 \%$ reduction in mortality

\footnotetext{
* Correspondence: heather.jaspan@sbri.org

'Children's Infectious Diseases Clinical Research Unit, Department of Paediatrics and Child Health and Centre for Infectious Diseases, Faculty of Health Sciences, Stellenbosch University, Tygerberg, South Africa Full list of author information is available at the end of the article
}

versus the prevailing standard of care at the time (the WHO 2006 guidelines) [7]. Importantly, most deaths occurred in the first few months of life and none of the infants had a CD $4<25 \%$ or advanced HIV disease according to Centers for Diseases Control criteria [4].

Diagnostic HIV-1 DNA PCR is recommended at 6 weeks of age in HIV-exposed infants going through Vertical Transmission Prevention (VTP) programs [8]. Although qualitative HIV-1 PCR testing is becoming more available, turn-around time for results and subsequent initiation of ART can take weeks. Clinical algorithms for HIV diagnosis in young infants could be useful for identifying those with probable HIV infection 
[9-13]. Interventions might be implemented for fasttracking early diagnosis.

Clinical algorithms for pediatric HIV diagnosis [9-13] have been developed but very few have used data on clinical manifestations from very young infants. To our knowledge, only one algorithm for this age group has been developed in Zimbabwe [9], using data from the pre-VTP era. Our aim was to investigate the predictive value of clinical characteristics for HIV infection in HIV-infected and HIV-exposed uninfected infants and to develop a clinical algorithm for identification of HIV infection in young infants who have failed VTP interventions.

\section{Methods}

\section{Participants, data collection and laboratory methods}

Data were collected during the screening phase of two clinical trials between 2005 to 2007 in Cape Town and Soweto, South Africa; the CHER trial, and a parallel observational cohort study. For randomization in the CHER study, baseline CD4 had to be $\geq 25 \%$ [4]. A small number of infants with lower CD4 percentage were enrolled in the parallel observation cohort, so were included in the present analysis. Infants were identified through VTP and referred to the study sites. VTP consisted of single dose nevirapine (NVP) to mother and newborn; and in Cape Town, Zidovudine (ZDV) was given to mothers from 32 weeks gestation, and to the infant for one week. HIV-exposed infants were tested by HIV-1 DNA PCR (Roche Amplicor HIV-1 DNA assay version 1.5, Roche Molecular Systems, Inc., Branchburg, $\mathrm{NJ}$ ) between 4 and 10 weeks of age. At screening, clinical signs were recorded and HIV infection was confirmed by plasma HIV RNA level > 5000 copies per ml (Roche Ampliprep/Cobas Amplicor assay, Roche Molecular Systems, Inc., Branchburg, NJ) (see below). High viral loads were not titrated beyond 750,000 viral copies/mL $(\log 5.88)$. HIV-exposed infants testing HIV-1 DNA PCR negative were still eligible for the cohort study, and were included in this analysis. HIV-infected infants had lymphocyte subsets measured using Beckman Coulter single platform lyse-no wash procedure using Immunoprep ${ }^{\mathrm{TM}}$ reagents with Flow Count ${ }^{\mathrm{TM}}$ fluorospheres. A small number of HIV-infected infants with CD $4 \%$ below 25\% were eligible for the concurrent cohort study and were therefore included in the analysis.

Data from screening for both studies were collected on predefined questionnaires by doctors aware of the infants' HIV status, but unaware of their degree of immunosupression. Reflux was defined as excessive vomiting after feeds. Hepatomegaly was defined as a palpable liver $>1 \mathrm{~cm}$ below the costal margin in the right mid-clavicular line. Splenomegaly was noted if the spleen was palpable. Encephalopathy was defined as symmetrically abnormal neurological examination, microcephaly or lack of head circumference growth, or cerebral atrophy on CT scan. Lymphadenopathy was defined as palpable lymph nodes greater than $1 \mathrm{~cm}$ in diameter. Lymphadenopathy was generalised if it was palpable in two or more areas including cervical, axillary, or inguinal regions. Diaper dermatitis was an abnormal rash of any type in the diaper area, candidal or otherwise. Standard World Health Organisation growth curves (available at http://www.who.int/childgrowth/standards/en/) were used to define cut-off values for $3^{\text {rd }}, 15^{\text {th }}$, and $50^{\text {th }}$ centiles (marked on the charts) for weight-for-age. Anemia and neutropenia were defined according to the Division of AIDS toxicity table for grading severity of Pediatric ( $\leq 3$ months of age) adverse experiences (available at http://rsc.tech-res.com/ safetyandpharmacovigilance/gradingtables.aspx)

\section{Statistical methods}

Stata version 9.0 (College Station, Texas) was used for analysis. Screening data were compared using KruskalWallis tests, analysis of variance (ANOVA), and Fisher's exact tests. Similar analysis was performed for CD $4 \%$ data. Multivariate regression was performed including age as a dependent variable to determine the relationship between log viral load and clinical findings. We estimated sensitivity, specificity, positive likelihood ratios, and age-adjusted odds ratios (OR), for the association between HIV status and clinical parameters. The 95\% confidence intervals $(\mathrm{CI})$ were calculated using standard methods. Receiver Operating Characteristic (ROC) curves were examined using combinations of clinical predictors of HIV infection. Characteristics with significant age-adjusted OR in univariate analyses $(\mathrm{p}<$ 0.05 ) were combined to achieve the highest Area Under the Curve (AUC) for the ROC.

\section{Ethical Considerations}

The Ethics Committees of the Universities of Stellenbosch and Witwatersrand approved the studies. Written informed consent was obtained from legal guardians of all infants.

\section{Results}

417 HIV-infected and 125 HIV-exposed, uninfected (HEU) infants were included. The median age at screening was 46 days (Interquartile range, [IQR] 38-55). The median CD $4 \%$ of the HIV-infected infants was $34 \%$ (IQR 28-41). Sixty-two HIV-infected infants with CD4 < $25 \%$ were included in the analysis.

Only $12 \%$ of infants were ever breastfed; uptake of VTP was $90 \%$. The demographics and prevalence of selected clinical characteristics of infants by infection status at screening are shown in Table 1 . Weight-for- 
Table 1 Frequency of selected physical findings and clinical events in very young HIV-infected and exposed uninfected infants.

\begin{tabular}{|c|c|c|c|c|}
\hline & Exposed uninfected $n=125$ & $\begin{array}{l}\text { Infected } \\
\mathrm{n}=417\end{array}$ & Unadjusted $\mathrm{p}$-value ${ }^{3}$ & Adjusted Odds Ratio $(95 \% \mathrm{Cl})^{3}$ \\
\hline $\begin{array}{l}\text { Female gender (\%) } \\
\text { Median age, days (IQR) }\end{array}$ & $\begin{array}{l}57(46) \\
48(38-51)\end{array}$ & $\begin{array}{l}239(57) \\
45(38-56)\end{array}$ & $\begin{array}{l}0.18 \\
0.07^{1}\end{array}$ & \\
\hline \multicolumn{5}{|l|}{ Anthropometry at screening } \\
\hline Median weight, kg (IQR) & $4.8(4.3-5.2)$ & $4.4(3.9-4.9)$ & $<0.001^{2}$ & $0.51(0.38-0.68)$ \\
\hline Weight $<3^{\text {rd }}$ centile $(\%)$ & $8(6.4)$ & $79(18.9)$ & $<0.001$ & $3.2(1.4-6.8)$ \\
\hline Weight $<15^{\text {th }}$ centile $(\%)$ & $23(18.4)$ & $166(39.8)$ & $<0.001$ & $2.8(1.7-4.6)$ \\
\hline Weight $<50^{\text {th }}$ centile $(\%)$ & $79(63.2)$ & $303(72.7)$ & 0.04 & $1.5(0.98-2.3)$ \\
\hline Median length, cm & $55(53-56)$ & $54(52-56)$ & $0.78^{2}$ & $0.97(0.91-1.04)$ \\
\hline Median $\mathrm{HC}, \mathrm{cm}$ & $39(38.4-40)$ & $39(38-40)$ & $0.20^{2}$ & $0.95(0.88-1.03)$ \\
\hline \multicolumn{5}{|c|}{ Physical exam findings at screening } \\
\hline Any LAD" $(\%)$ & $6(5)$ & $132(32)$ & $<0.001$ & $8.8(3.8-20.9)$ \\
\hline Candidal rash (\%) & $8(6)$ & $46(11)$ & 0.17 & $1.6(0.8-3.6)$ \\
\hline Atopic dermatitis (\%) & $13(10)$ & $83(20)$ & 0.01 & $2.0(1.1-3.7)$ \\
\hline Diaper dermatitis & $23(18)$ & $149(36)$ & $<0.001$ & $2.4(1.5-4.0)$ \\
\hline Oral thrush (\%) & $13(10)$ & $163(39)$ & $<0.001$ & $5.6(3.0-10.2)$ \\
\hline Hepatomegaly (\%) & $1(1)$ & $70(17)$ & $<0.001$ & $23.5(3.2-170.8)$ \\
\hline Splenomegaly (\%) & $0(0)$ & $30(7)$ & $<0.001$ & * \\
\hline \multicolumn{5}{|c|}{ Clinical event prior to or at screening } \\
\hline Gastroenteritis (\%) & $8(6)$ & $44(11)$ & 0.17 & $1.7(0.8-3.7)$ \\
\hline Otitis media (\%) & $4(3)$ & $6(1)$ & 0.20 & $0.4(0.1-1.5)$ \\
\hline Sepsis (\%) & $3(2)$ & $12(3)$ & 1.00 & $1.0(0.3-3.7)$ \\
\hline URTI (\%) & $24(19)$ & $81(19)$ & 1.00 & $1.0(0.6-1.7)$ \\
\hline Reflux (\%) & $0(0)$ & $15(4)$ & 0.03 & * \\
\hline Pneumonia (\%) & $7(6)$ & $29(7)$ & 0.67 & $1.2(0.5-2.8)$ \\
\hline \multicolumn{5}{|l|}{ Laboratory findings } \\
\hline Anemia (\%) & $0(0)$ & $22(5)$ & 0.004 & * \\
\hline Neutropenia (\%) & $0(0)$ & $15(4)$ & 0.03 & * \\
\hline
\end{tabular}

${ }^{1}$ Kruskal Wallis ${ }^{2}$ ANOVA ${ }^{3}$ Fishers exact unless otherwise specified

${ }^{3}$ Age-adjusted OR of infected infants versus HIV exposed-uninfected infants

IQR - Interquartile range. Cl - Confidence Interval. HC - Head circumference. URTI - Upper respiratory tract infection.

\#Any lymphadenopathy - includes generalized, cervical, axillary or inguinal

*Small sample sizes prevented the calculation of adjusted OR

age of HIV-infected infants was significantly lower than in HIV-exposed uninfected infants. Infected infants were more likely to have weight below the $3^{\text {rd }}$ and $15^{\text {th }}$ but not the $50^{\text {th }}$ centile. Lymphadenopathy (axillary, cervical, generalised, or any), oral thrush, splenomegaly, or hepatomegaly and a history of reflux were more common in infected infants. Diaper dermatitis (candidal, atopic, or other) was also significantly associated with HIV status. Both anemia and neutropenia of grade 2 or above were almost exclusively found in HIV-infected infants. Electrolyte and liver function test results did not differ between the groups (data not shown). However, $27 \%$ of HIV-infected infants had weight above the $50^{\text {th }}$ centile.

The 62 HIV-infected infants with CD4\% < 25 had a median age of 51.5 days [IQR 42-66]. Their median CD4\% was 20.9 [IQR 17.6-23.3]. Compared with those with CD4 $\geq 25 \%$, severely immunocompromised infants were older and more likely to have weight below $3^{\text {rd }}$ and $15^{\text {th }}$ centile for age, but not $50^{\text {th }}$ (Table 2). Severely immunosuppressed infants were also more likely to have lymphadenopathy, hepatomegaly, splenomegaly, and a history of gastroenteritis or pneumonia.

The median log viral load of the HIV-infected infants was 5.88 [Range 3.78-5.88]. In a univariate regression model, there was weak association between log viral load and age (regression coefficient 2.77; 95\% CI -0.53 6.06). No age-adjusted clinical finding or event was predictive of higher viral load except oral thrush (regression coefficient 0.09, 95\% CI 0.02; 0.16).

The sensitivity, specificity, and positive likelihood ratio $(\mathrm{LR}+)$ of selected findings for HIV infection is shown in Table 3. Low weight-for-age, any lymphadenopathy, hepatomegaly, splenomegaly, oral thrush, and reflux were all highly specific but insensitive for HIV infection. 
Table 2 Frequency of selected physical findings and clinical events in very young HIV-infected with CD4\% $<25 \%$ (severe immune suppression) versus CD4 $\geq 25 \%$

\begin{tabular}{|c|c|c|c|c|}
\hline & CD4 $<25 \% n=62$ & $\mathrm{CD} 4 \geq 25 \% \mathrm{n}=313$ & Unadjusted $p$-value ${ }^{3}$ & AOR $(95 \% \mathrm{Cl})^{3}$ \\
\hline Female gender (\%) & $29(46.8)$ & $187(59.7)$ & 0.06 & \\
\hline Median age, days (IQR) & $52(42-66)$ & $45(39-55)$ & $0.002^{1}$ & \\
\hline \multicolumn{5}{|l|}{ Anthropometry at screening } \\
\hline Median weight, kg (IQR) & $4.3(3.9-5)$ & $4.4(4.0-4.9)$ & 0.228 & $1.6(1.1-2.4)$ \\
\hline Weight $<3^{\text {rd }}$ centile $(\%)$ & 19 (30.6) & $52(16.6)$ & 0.010 & NA \\
\hline Weight $<15^{\text {th }}$ centile (\%) & $36(56.4)$ & $115(37.0)$ & 0.004 & NA \\
\hline Weight $<50^{\text {th }}$ centile (\%) & $51(82)$ & $228(72.8)$ & 0.121 & NA \\
\hline Median length, cm & $54(52.5-55)$ & $54(52-56)$ & 0.563 & $1.1(0.98-1.2)$ \\
\hline Median $\mathrm{HC}, \mathrm{cm}$ & $39(38-40)$ & $39(38-40)$ & 0.859 & $1.1(0.9-1.1)$ \\
\hline \multicolumn{5}{|c|}{ Physical exam findings at screening } \\
\hline Any LAD\# (\%) & $33(53.2)$ & $93(29.7)$ & $<0.001$ & $0.4(0.2-0.7)$ \\
\hline Atopic dermatitis (\%) & $14(22.6)$ & $64(20.4)$ & 0.705 & $1.1(0.5-2.1)$ \\
\hline Diaper dermatitis & $28(45.2)$ & $111(35.5)$ & 0.149 & $0.7(0.4-1.2)$ \\
\hline Oral thrush (\%) & $29(46.8)$ & $115(36.7)$ & 0.138 & $0.7(0.4-1.1)$ \\
\hline Hepatomegaly (\%) & $11(17.7)$ & $19(6.1)$ & 0.002 & $0.4(0.2-0.8)$ \\
\hline Splenomegaly (\%) & 19 (30.6) & $51(16.3)$ & 0.008 & $0.5(0.3-0.9)$ \\
\hline \multicolumn{5}{|c|}{ Clinical event prior to or at screening } \\
\hline Gastroenteritis (\%) & $12(19.4)$ & $29(9.3)$ & 0.020 & $0.5(0.2-1.0)$ \\
\hline Otitis media (\%) & $1(1.6)$ & $5(1.6)$ & 0.993 & $1.5(0.2-13.8)$ \\
\hline Sepsis (\%) & $4(6.5)$ & $7(2.2)$ & 0.072 & $0.4(0.1-1.5)$ \\
\hline URTI (\%) & $11(17.7)$ & $65(20.8)$ & 0.558 & $1.2(0.6-2.5)$ \\
\hline Reflux (\%) & $3(4.8)$ & $11(35.1)$ & 0.615 & $0.6(0.2-2.4)$ \\
\hline Pneumonia (\%) & $11(17.2)$ & $17(5.4)$ & 0.001 & $0.2(0.1-0.6)$ \\
\hline \multicolumn{5}{|l|}{ Laboratory findings } \\
\hline Anemia (\%) & $4(6.5)$ & $18(5.8)$ & 0.830 & $1.0(0.3-3.0)$ \\
\hline Neutropenia (\%) & $4(6.5)$ & $9(2.9)$ & 0.160 & $0.4(0.1-1.2)$ \\
\hline
\end{tabular}

${ }^{1}$ Kruskal Wallis ${ }^{2}$ ANOVA ${ }^{3}$ Fishers exact unless otherwise specified

${ }^{3}$ Age-adjusted OR of HIV-infected infants with CD4 $\geq 25 \%$ versus those with CD4 $<24 \%$

IQR - Interquartile range. Cl - Confidence Interval. HC - Head circumference. URTI - Upper respiratory tract infection.

\#Any lymphadenopathy - includes generalized, cervical, axillary or inguinal

*Small sample sizes prevented the calculation of adjusted OR

Excluding infants with weight above the $50^{\text {th }}$ centile, as in the algorithm by Iliff et al [9] improved sensitivity and specificity.

A combination of various clinical findings in a ROC improved sensitivity without compromising specificity (Table 4). For example, if any one of six clinical findings - oral thrush, hepatomegaly, splenomegaly, lymphadenopathy, diaper dermatitis, or weight below the $15^{\text {th }}$ centile were present, the sensitivity for HIV infection amongst HIV-exposed infants was $78 \%$ and the specificity $62 \%$. Increasing the weight cut-off to $<50^{\text {th }}$ centile improved sensitivity to $89 \%$ (Table 4 ). By excluding diaper dermatitis, including reflux, and changing the weight cut-off to below the $3^{\text {rd }}$ centile, specificity increased to $80 \%$ but sensitivity decreased to $64 \%$. When cut-offs to any two findings were increased, specificity approached 100\%, but compromised sensitivity. Although nurses can be trained to recognise hepatosplenomegaly and lymphadenopathy, simple algorithms may be preferred by programs with high-volume or nurse-run programs. Table 4 shows simplified algorithms including only diaper dermatitis, oral thrush, and weight $<3^{\text {rd }}$ and $50^{\text {th }}$ centiles, giving sensitivities ranging from 61 to as high as $85 \%$, with that for $15^{\text {th }}$ centile falling somewhere in between (data not shown).

When these algorithms were applied to HIV-infected infants to predict severe immune suppression (CD4 < $25 \%$ ), the algorithms were sensitive, but poorly specific using one cut-off finding. For example, any one of the six clinical findings in Table $4 \mathrm{~B}$ (weight below $50^{\text {th }}$ centile, hepatomegaly, splenomegaly, diaper dermatitis, and lymphadenopathy) had a sensitivity of $89 \%$ but specificity of $2 \%$ for predicting severe immune suppression.

\section{Discussion}

We found that certain clinical signs and symptoms are highly specific for HIV infection in relatively healthy infants exposed to ARVs for prevention of vertical 
Table 3 Sensitivity and specificity of select clinical findings and events for distinguishing HIV-infected from exposed uninfected infants

\begin{tabular}{llll}
\hline Characteristic & All exposed infants & & Specificity $(\mathbf{9 5 \%} \mathbf{C l})$ \\
\hline General LAD & Sensitivity $(\mathbf{9 5} \% \mathbf{C l})$ & $97.6(93.1-99.5)$ & 6.1 \\
Any LAD & $14.6(11.8-17.9)$ & $95.2(89.8-98.2)$ & 4.8 \\
Hepatomegaly & $24.4(20.9-28.3)$ & $99.2(95.6-100)$ & 13 \\
Splenomegaly & $13.0(10.2-16.1)$ & 100 & $n / a$ \\
Oral thrush & $5.6(3.8-7.8)$ & $89.6(82.8-94.3)$ & 164 \\
Diaper rash & $30.2(26.3-34.2)$ & $81.6(12-26.3)$ & 1.9 \\
Reflux & $35.7(31.1-40.5)$ & 100 & $n / a$ \\
Weight $<3^{\text {rd }}$ & $2.8(1.6-2.5)$ & $93.6(87.8-97.2)$ & 2.3 \\
Weight $<15^{\text {th }}$ & $14.6(11.7-17.9)$ & $81.6(73.7-87.9)$ & 1.7 \\
\hline
\end{tabular}

WAZ - weight-for-age score. $\mathrm{Cl}$ - confidence interval.

+LR - positive likelihood ratio. n/a - not applicable.

transmission below 10 weeks of age. Any one of a complex of findings including oral thrush, lymphadenopathy, hepatomegaly, splenomegaly, weight centile, diaper dermatitis, or reflux, give specificities and sensitivities of up to $89 \%$ and $80 \%$ respectively. A small number of studies prospectively investigated clinical symptoms in acutely infected infants during breastfeeding. In a study by Rouet et al in Cote d'Ivoire, 22 of 342 infants developed postnatal HIV infection. Acute retroviral syndrome, generalised lymphadenopathy and dermatitis were predictive of acute HIV infection [14]. Richardson et al performed a similar study in Kenya where 40 (71\%) of acutely infected infants were below 2 months of age in a cohort of 189 infants. Acute infection was associated with lymphadenopathy in $\geq 2$ sites and upper respiratory tract infection [15].

In a larger study from the pre-VTP era of infants born to HIV-infected women, the Zimbabwe Vitamin A for Mothers and Babies (ZVITAMBO) group evaluated WHO, South African, and Zimbabwean algorithms for HIV diagnosis in 6-week-old infants [9]. In this study, $52 \%$ of HIV-infected infants had a weight for age $<50^{\text {th }}$ centile ( $Z$ score $\leq 0)$, and by focussing on this group, the author's developed diagnostic algorithms based on clinical signs with relatively high yield. However, by focussing only on HIV-exposed infants with weight-forage below the median, sensitivity of their clinical algorithm for HIV diagnosis was $65 \%$. When combined with

Table 4 Receiver Operating Characteristic (ROC) curves of combinations of clinical findings with the greatest area under the curve (AUC), and sensitivity and specificity of various cut-off numbers of findings in predicting HIVinfection among exposed infants.

\begin{tabular}{|c|c|c|c|c|}
\hline $\begin{array}{l}\text { One of a combination of oral thrush, any lymphadenopathy, hepatomegaly, } \\
\text { splenomegaly, plus: }\end{array}$ & $\begin{array}{l}\text { ROC } \\
\text { AUC }\end{array}$ & $\begin{array}{l}\text { Findings } \\
\text { present }\end{array}$ & $\begin{array}{l}\text { Sensitivity } \\
(\%)\end{array}$ & $\begin{array}{l}\text { Specificity } \\
(\%)\end{array}$ \\
\hline \multirow[t]{3}{*}{ A: weight $<15$ th centile, diaper dermatitis } & 0.754 & 1 & 77.9 & 61.2 \\
\hline & & 2 & 47.7 & 88.0 \\
\hline & & 3 & 25.2 & 98.4 \\
\hline \multirow[t]{3}{*}{ B: weight < 50th centile, diaper dermatitis } & 0.717 & 1 & 88.9 & 29.6 \\
\hline & & 2 & 48 & 90.4 \\
\hline & & 3 & 20.6 & 99.2 \\
\hline \multirow[t]{3}{*}{ C: weight $<3$ rd centile, reflux } & 0.744 & 1 & 63.8 & 80 \\
\hline & & 2 & 31.4 & 97.6 \\
\hline & & 3 & 12.5 & 100 \\
\hline One of a combination of oral thrush, diaper dermatitis, plus: & $\begin{array}{l}\text { ROC } \\
\text { AUC }\end{array}$ & $\begin{array}{l}\text { Findings } \\
\text { present }\end{array}$ & $\begin{array}{l}\text { Sensitivity } \\
(\%)\end{array}$ & $\begin{array}{l}\text { Specificity } \\
(\%)\end{array}$ \\
\hline \multirow[t]{3}{*}{ D: weight $<3^{\text {rd }}$ centile } & 0.682 & 1 & 60.7 & 72 \\
\hline & & 2 & 28.3 & 92.8 \\
\hline & & 3 & 4.8 & 100 \\
\hline \multirow[t]{2}{*}{ E: weight $<50^{\text {th }}$ centile } & 0.666 & 1 & 84.7 & 30.4 \\
\hline & & 2 & 46.8 & 82.4 \\
\hline
\end{tabular}


$\geq$ two other physical signs (e.g. pneumonia, ear discharge, treatment for TB, oral candidiasis), the sensitivity decreased to $20 \%$ but the specificity increased to $94 \%$. Our algorithm including weight $<50^{\text {th }}$ centile (algorithm B; table 3) but with different findings improved sensitivity to $86 \%$ in this age group. Additionally, a simplified algorithm that included weight $<50^{\text {th }}$ maintained high sensitivity of $85 \%$. However, algorithms of this sort exclude the high proportion of HIV-infected infants with weight above $50^{\text {th }}$ centile.

The ZVITAMBO approach [9] of focusing where yield for subsequent DNA PCR testing may be highest appears useful in settings with severe resource limitations [16]. South Africa is a mid-income country that can afford HIV-1 diagnostic PCRs on all HIV-exposed infants. Therefore, although limiting tests to infants below the $50^{\text {th }}$ centile may reduce costs, the number of infants missed will be unacceptably high. Consequently, an algorithm with higher sensitivity would be useful. Additionally, in settings where PCR is unavailable or turnaround time for results unacceptable, an algorithm with high sensitivity may be preferred as a strategy for ART initiation to prevent the early infant mortality associated with HIV infection. Therefore using algorithm B, may be preferable in these settings. The advantage of algorithms such as ours is that programs could adapt the cut-off to suit their needs.

To our knowledge, ours is the first study to investigate diagnostic algorithms of predominantly formula fed infants in the VTP era. Our algorithms have the highest sensitivity for HIV identification of all those previously published, particularly in this age group.

The majority of other algorithms and guidelines to identify HIV-infected children have not included infants below two months of age [10-12]. Jones et al found a sensitivity of $17 \%$ when applying the Integrated Management of Childhood Illness (IMCI) algorithms for predicting pediatric HIV infection to a cohort of 301 HIVexposed infants in VTP programs, of whom 26 (8.7\%) were shown to be HIV-infected by HIV DNA PCR [10]. However, using clinical signs similar to those selected in our study (hepatosplenomegaly, recurrent infections, failure to thrive, and candidiasis) and where doctors evaluating the infants were unaware of infection status, the sensitivity for HIV infection increased to $56 \%$ at 6 weeks of age [10].

As expected, increasing the number of positive signs reduces sensitivity but increases specificity. Reflux occurred at low frequency (3.4\%) but appeared very specific, albeit difficult to define. Of note, 11 (73\%) of the 15 infants with reflux also had oral thrush, which could have aggravated or caused the symptom; swallowing incoordination, an early sign of encephalopathy, could also have contributed. That oral thrush and low weight for age were so highly specific in this population suggests that these findings cannot only be attributed to formula feeding or co-morbidities such as malnutrition and TB.

A limitation of our study is that clinician's knowledge of the infants' HIV status may have influenced the finding and reporting of clinical findings. On the other hand, HIV-infected infants eligible for CHER were selected for lack of severe clinical disease [4] and doctors trained for the study evaluated all infants. Our results require validation in other settings, for example, where malaria and breastfeeding are prevalent, and in VTP programs with larger numbers of HIV-exposed uninfected infants and with lower HIV prevalence. Our study included a large group of HIV-infected infants (77\%) and a smaller number of HIV-exposed infants, all below 10 weeks of age, in contrast to all of the other studies. Also, as mentioned, infants with severe clinical disease, where the diagnosis may be more obvious, were also excluded. In settings where this algorithm would be useful, the prevalence of HIV-infection amongst exposed infants would be lower, and thus affect the positive and negative predictive values of the algorithm. In addition, due to selection methods, most HIV-infected infants included in this analysis were relatively well, with a median CD4 count of $34 \%$. The algorithm may perform better in a general population of HIV-infected infants. Additionally, only $12 \%$ of this cohort was breastfeeding. In conclusion, a combination of clinical findings in infants below 10 weeks of age may be useful for predicting HIV infection and prioritising care. There is urgent need to validate these findings in other settings and to determine their relevance in diverse settings.

\section{Conclusion}

A combination of physical findings is helpful in identifying infants most likely to be HIV-infected. This may inform management algorithms and provide guidance for focused laboratory testing or antiretroviral initiation in some settings, and should be further validated in these settings and elsewhere.

\section{List of Abbreviations}

HIV: Human immunodeficiency virus; CHER: Children with HIV Early Antiretroviral; WHO: World Health Organization; ART: Antiretroviral therapy; VTP: Vertical transmission prevention; DNA: Deoxyribonucleic acid; PCR: Polymerase chain reaction; NVP: Nevirapine; ZDV: Zidovudine; OR: Odds ratio; IQR: Interquartile range; ANOVA: Analysis of variance; LR: Likelihood ratio; IMCl: Integrated Management of Childhood Illness

\section{Acknowledgements and funding}

Support for this study was provided by the National Institute of Allergy and Infectious Diseases (NIAID) of the US National Institutes for Health (NIH), through the Comprehensive International Program of Research on AIDS (CIPRA) network, grant number U19 Al53217. The Departments of Health of the Western Cape and Gauteng, South Africa provided additional support. The content of this publication does not necessarily reflect the views or 
policies of NIAID, nor does mention of trade names, commercial projects, or organizations imply endorsement by the US Government. The study was also conducted as an Investigational New Drug (IND) Number: IND 71,494 under the supervision of the Food and Drug Administration.

We would like to thank the families and children who participated in the trial, the healthcare workers at peripheral clinics, and the following individuals: Helena Rabie, Anita Janse van Rensburg, Els Dobbels, George Fourie, Marietjie Bester, Wilma Orange, Ronelle Arendze, Catherine Andrea, Marlize Smuts, Kurt Smith, Theresa Louw, Alec Abrahams, Kenny Kelly, Amelia Bohle, Irene Mong, Jodie Howard, Tanya Cyster, Genevieve Solomon, Galroy Benjamin, Jennifer Mkalipi, Edward Barnes (Children's Infectious Diseases Clinical Research Unit), Wilma Pelser, Ravindre Panchia, Handre Truter, Valerie Khemese, Sibongile Sithebe, Refilwe Khumalo, Ronelle van Niekerk, and Jan Steyn of the Perinatal HIV research unit. United Kingdom: Abdel G Babiker (Medical Research Council, Clinical Trials Unit, London) JeanMarc Steens, Wendy $\times$ Snowden, Eddie Loeliger, Navdeep Thoofer (GlaxoSmithKline).

\section{Author details}

${ }^{1}$ Children's Infectious Diseases Clinical Research Unit, Department of Paediatrics and Child Health and Centre for Infectious Diseases, Faculty of Health Sciences, Stellenbosch University, Tygerberg, South Africa. ${ }^{2}$ School of Child and Adolescent Health, University of Cape Town, Cape Town, South Africa. ${ }^{3}$ Children's Hospital and Regional Medical Centre, Department of Pediatrics, University of Washington, Seattle, WA, USA. ${ }^{4}$ Infectious Diseases Epidemiology Unit, School of Public Health \& Family Medicine, University of Cape Town, Cape Town, South Africa \& Department of Epidemiology, Mailman School of Public Health, Columbia University, USA. ${ }^{5}$ Department of Science and Technology/National Research Foundation: Vaccine Preventable Diseases; \& MRC/WITS Respiratory and Meningeal Pathogens Research Unit; University of the Witwatersrand, Johannesburg, South Africa. ${ }^{6}$ Perinatal HIV research Unit, University of the Witwatersrand, Johannesburg, South Africa. ${ }^{7}$ Medical Research Council, Clinical Trials Unit, London. UK. ${ }^{8}$ Department of Molecular Medicine and Haematology, University of the Witwatersrand and National Health Laboratory Service, Johannesburg, South Africa. ${ }^{9}$ Seattle Biomedical Research Institute, 307 Westlake Ave S, Seattle WA 98109 or University of Cape Town Health Sciences Faculty, Anzio Rd Observatory, Cape Town 7925, South Africa.

\section{Authors' contributions}

All authors read and approved the final manuscript. HBJ performed primary data analysis and was primary manuscript author; LM assisted with data analysis and manuscript development, and overall study design; SAM developed the cohorts and edited the manuscript; AV developed the cohorts and edited the manuscript; DMG provided scientific advice, clinical advice, and manuscript edits; WSS provided laboratory support and manuscript edits; ED contributed to clinical management of the cohort and was the primary clinical advisor; MFC provided the design and contributed to manuscript authoring.

\section{Competing interests}

The authors declare that they have no competing interests.

Received: 26 April 2011 Accepted: 21 November 2011 Published: 21 November 2011

\section{References}

1. Newell ML, Brahmbhatt H, Ghys PD: Child mortality and HIV infection in Africa: a review. AIDS 2004, 18:S27-S34.

2. Newell ML, Coovadia H, Cortina-Borja M, Rollins N, Gaillard P, Dabis F, Ghent International AIDS Society (IAS) Working Group on HIV infection in Women and Children: Mortality of infected and uninfected infants born to HIV-infected mothers in Africa: a pooled analysis. Lancet 2004, 364:1236-1243.

3. Brahmbhatt H, Kigozi G, Wabwire-Mangen F, Serwadda D, Nalugoda F, Sewankambo N, Lutalo T, Kidduggavu M, Wawer M, Gray R: Mortality in HIV-infected and uninfected children of HIV-infected and uninfected mothers in rural Uganda. J Acquir Immune Defic Syndr 2006, 41:504-508.

4. Violari A, Cotton M, Gibb D, Babiker AG, Steyn J, Madhi SA, Jean-Philippe P, McIntyre JA, The CHER Study Team: ART initiated before 12 weeks reduces early mortality in young HIV-infected infants: evidence from the
Children with HIV Early Antiretroviral Therapy (CHER) Study. N Engl I Med 2008, 358:2233-2244.

5. McNally LM, Jeena PM, Gajee K, Thula S, Sturm AW, Cassol S, Tomkins AM, Coovadia AH, Goldblatt D: Effect of age, polymicrobial disease, and maternal HIV status on treatment response and cause of severe pneumonia in South African children: a prospective descriptive study. Lancet 2007, 369:1440-1451.

6. Dunn DT, HIV Paediatric Prognostic Markers Collaborative Study: Short-term risk of disease progression in HIV-1-infected children receiving no antiretroviral therapy or zidovudine monotherapy: a meta-analysis. Lancet 2003, 362:1605-1611.

7. World Health Organisation: Antiretroviral therapy of HIV infection in infants and children in resource-limited settings: towards universal access Geneva; 2006.

8. World Health Organisation: Report of the WHO Technical Reference Group, Paediatric HIV/ART Care Guideline Reference Group Meeting Geneva, Switzerland; 2008.

9. Iliff PJ, Ntozini R, Nathoo KJ, Piwoz E, Moulton L, ZVITAMBO Study: Making a working clinical diagnosis of HIV infection in infants in Zimbabwe. Trop Med Int Health 2008, 13:1459-1469.

10. Jones SA, Sherman GG, Coovadia AH: Can clinical algorithms deliver an accurate diagnosis of HIV infection in infancy? Bull World Health Organ 2005, 83:559-560.

11. Chintu C, Malek A, Nyumbu M, Luo C, Masona J, DuPost HL, Zumla A: Case definitions for paediatric AIDS: the Zambian experience. Int J STD AIDS 1993, 4:83-85.

12. Lepage $P$, van de Perre $P$, Dabis $F$, Comenges D, Orbinski J, Hitimana DG, Bazubagira A, van Goethem C, Allen S, Butzler JP: Evaluation and simplification of the World Health Organization clinical case definition for paediatric AIDS. AIDS 1989, 3:221-225.

13. World Health Organisation: Report on the Workshop on Adaptation of IMCI Guidelines to include HIV/AIDS Harare, Zimbabwe; 2001 [http://www.who.int/ ], Accessed June 152008.

14. Rouet F, Elenga N, Msellati P, eMontcho C, Viho I, Sakaravitch C, Danel C, Rouzioux C, Leroy V, Dabis F, ANRS 049 Abidjan DITRAME Study: Primary HIV-1 infection in African children infected through breastfeeding. AIDS 2002, 16:2303-9.

15. Richardson BA, Nduati R, Mbori-Ngacha D, Overbaugh J, John-Stewart GC: Acute HIV infection among Kenyan Infants. Clin Infect Dis 2008, 46:289-95.

16. Sherman GG, Matsebula TC, Jones SA: Is early HIV testing of infants in poorly resourced prevention of mother to child transmission programmes unaffordable? Trop Med Int Health 2005, 10:1108-1113.

\section{Pre-publication history}

The pre-publication history for this paper can be accessed here: http://www.biomedcentral.com/1471-2431/11/104/prepub

doi:10.1186/1471-2431-11-104

Cite this article as: Jaspan et al:: Utility of clinical parameters to identify HIV infection in infants below ten weeks of age in South Africa: a prospective cohort study. BMC Pediatrics 2011 11:104.

\section{Submit your next manuscript to BioMed Central and take full advantage of:}

- Convenient online submission

- Thorough peer review

- No space constraints or color figure charges

- Immediate publication on acceptance

- Inclusion in PubMed, CAS, Scopus and Google Scholar

- Research which is freely available for redistribution

Submit your manuscript at www.biomedcentral.com/submit
C Biomed Central 